\title{
Determination of Surface Topography from Laser Ablation using EBSD
}

\author{
P.G. Callahan ${ }^{1}$, M.P. Echlin ${ }^{1}$, T.M. Pollock ${ }^{1}$, M. De Graef ${ }^{2}$ \\ ${ }^{1}$ Materials Department, University of California at Santa Barbara, Santa Barbara, CA 93106, \\ USA \\ 2 Department of Materials Science and Engineering, Carnegie Mellon University, Pittsburgh, \\ PA 15213, USA
}

In the last decade, serial sectioning with dual beam Focused Ion Beam-Scanning Electron Microscopes (FIB-SEM) has been used to successfully gather 3-D information from a variety of materials. The sizes of the 3-D volumes interrogated with this technique are limited by the milling rate of the Ga ion beams typically used. In order to collect larger volumes of data, a novel TriBeam system that combines ultrafast lasers with scanning electron microscopes has been developed [1]. The use of femtosecond laser ablation for machining allows for rapid machining of volumes of material approaching the $\mathrm{mm}^{3}$ scale. Machining with lasers results in a periodic surface roughness called the Laser-Induced Periodic Surface Structure (LIPSS); this surface roughness has a wavelength on the order of the wavelength of the machining laser [2]. A nickel sample was machined by laser ablation in the TriBeam system, and the resulting surface was scanned using electron backscatter diffraction (EBSD).

The method used to determine the surface topography uses data collected with an EBSD detector and is based on the fact that the location as well as intensity of the backscattered electrons is recorded on the EBSD detector. It is the location of the background intensity that is useful in determining the surface topography. It has been shown previously that the background intensity on an EBSD detector can be modeled using Monte Carlo simulations [3]; the backscattered electrons contributing to the background show nearly specular reflection from the sample surface. The deviation from specular reflection is a function of atomic number and incidence angle. This deviation was determined for $\mathrm{Ni}$ using MC simulations. A plot of the intensity profile of the backscatter electrons on the EBSD detector versus the incidence angle of the electron beam on a Ni sample is shown in Fig. 1. These intensity profiles were determined via simulation and can be used to determine the deviation from specular behavior. The location of maximum intensity of the backscatter electrons on the detector is determined, and can be used to determine the exit direction of the backscattered electrons. This exit direction, along with the geometry of the experimental setup and deviation from specular behavior indicate the components of local surface normal on the sample, $n_{x}=-\partial_{x} z$ and $n_{y}=-\partial_{y} z$. Next, the surface height function, or topography, is reconstructed from the local surface normals. The surface height function for the Ni sample is shown in Fig. 2.

References

[1] MP Echlin, M Straw, S Randolph, J Filevich and TM Pollock, Materials Characterization, 100, 1, 2015.

[2] JE Sipe, JF Young, JS Preston and HM van Driel, Physical Review B, 27, 1141, 1983.

[3] PG Callahan and M DeGraef, Microscopy and Microanalysis, 19, 1255, 2013. 


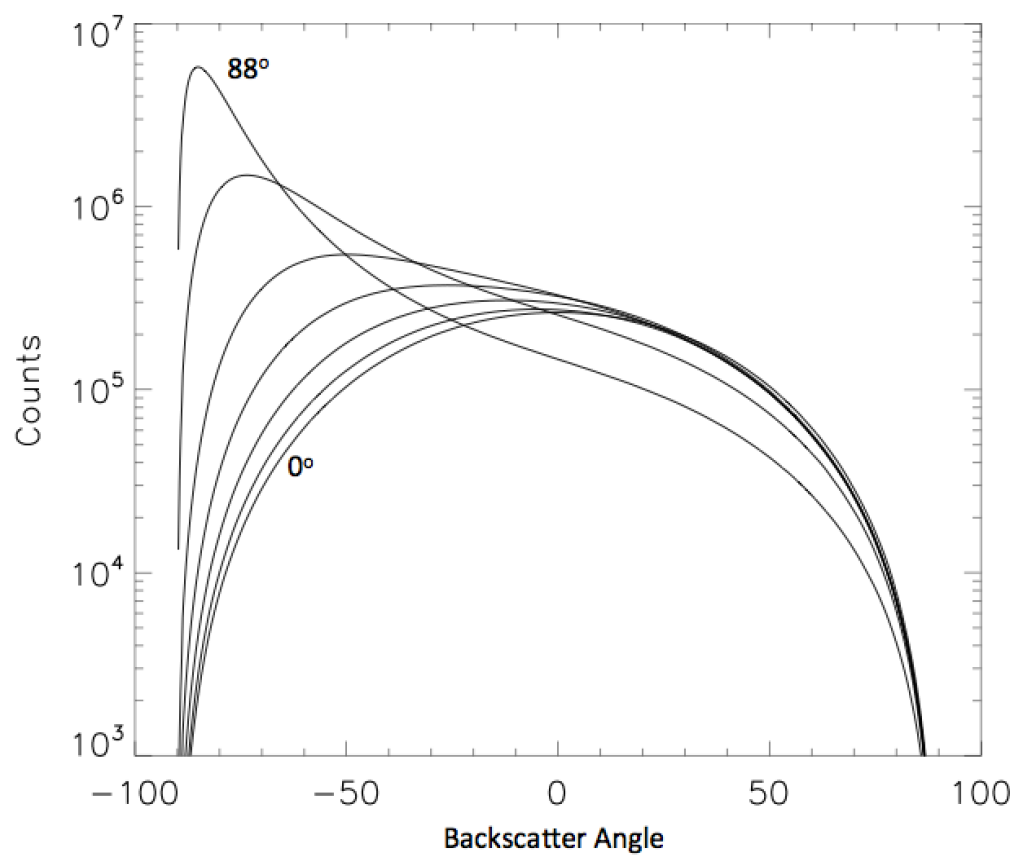

Figure 1: The intensity profiles of backscattered electrons for different incidence angles for incident electron energy of $30 \mathrm{keV}$ as determined from simulations. Increasing incidence angle corresponds to increased left skewness.

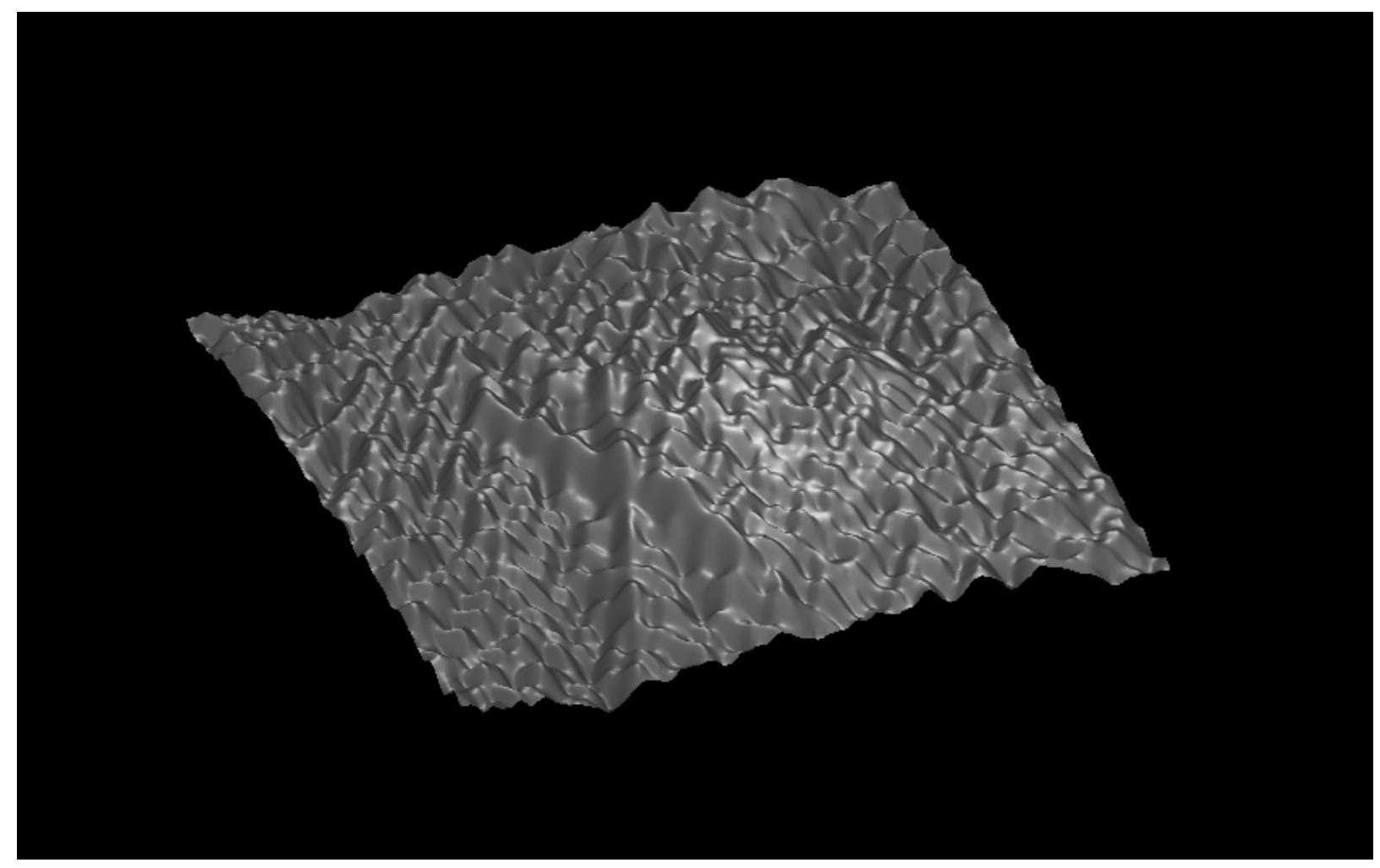

Figure 2: The surface height profile of the Ni sample as determined from EBSD data is shown. 